\title{
Study of tensile load carrying capacity of Iso -Truss with two different positions of longitudinal members
}

\author{
Mangesh S. Gavade ${ }^{*}$, S. Roy ${ }^{\ddagger}$, S. H. Barhatte ${ }^{\dagger}$ and S. S. Mujumdar ${ }^{\dagger}$ \\ ${ }^{\dagger}$ Department of Mechanical Engineering, Savitribai Phule Pune University, MIT College of Engineering, Pune, India \\ *Defence Research and Development Organization (R \&DE (E)), Pune, India .
}

Accepted 02 March 2016, Available online 15 March 2016, Special Issue-4 (March 2016)

\begin{abstract}
The paper discusses tensile load carrying capacity of an open lattice structure IsoTruss through finite element modeling and analysis. IsoTruss is Web like structure consisting of isosceles triangle within the geometry of its structure. IsoTruss provides a light weight structure when carbon fiber is used as the working material. The tensile strength of IsoTruss for two set of position of longitudinal members with respect to bay length is studied. The study concludes that IsoTruss with internal and external longitudinal members carry more tensile load compared to the IsoTruss with only internal longitudinal member. Also the Strength to Weight ratio for IsoTruss with internal and external longitudinal is higher than IsoTruss with only internal longitudinal members.
\end{abstract}

Keywords: IsoTruss, Bay length, Longitudinal member, tensile loading,Flexural behavior, strength to weight ratio, etc.

\section{Introduction}

IsoTruss is an open lattice structure. Open lattice structure is a systematic and periodic arrangement of points as observed in ions and molecules of elements. IsoTruss is a web like structure where the lattice points in the structure are attached in a specific manner to fulfill the desired design considerations. The open lattice synthesis of IsoTruss gives an opportunity for an ultralight weight structure. IsoTruss finds its applications in support structures of communication system over a satellite, bicycle frame, reinforced with concrete. It consists of longitudinal and helical members. Longitudinal members are aligned along the length of the IsoTruss and helical members spiral around the axis of IsoTruss in clockwise and anticlockwise directions. Axial loads are taken care by the longitudinal members. Helical members take care of the torsion and bending loads. Bay length is the distance between similar geometry within the structure. Outer diameter of IsoTruss is the maximum end to end distance of the cross section geometry. It can also be stated as the diameter of the circle enclosing the cross sectional geometry of IsoTruss. Figure 1 illustrates the various parameters of the IsoTruss. Tetrahedron is a pyramid formed due to the intersection of the clockwise and anticlockwise spiraling helical members. Longitudinal members passing through the base of the tetrahedron are

*Corresponding author: Mangesh S. Gavade termed as the internal longitudinal members and the one passing through the apex of the tetrahedron are termed as external longitudinal members.

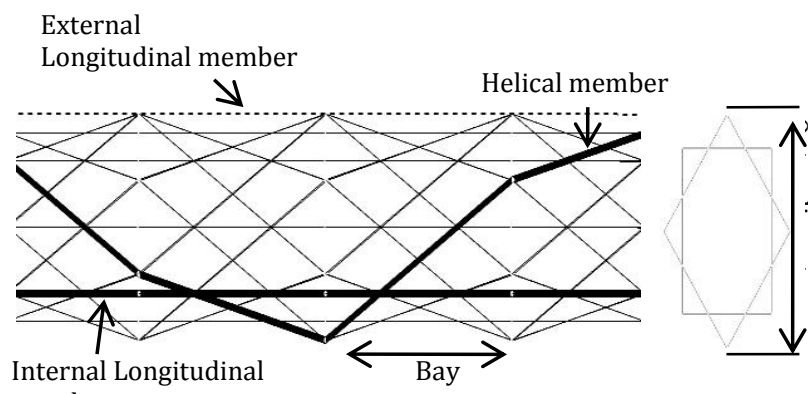
member

Fig.1 Parameters of 8 node configuration IsoTruss

Different configurations of IsoTruss are possible depending on the number of longitudinal or helical members. 6 node configuration represents a IsoTruss with 6 longitudinal members and 12 helical members, 6 spiraling clockwise and 6 spiraling anticlockwise around the longitudinal members. The cross section of 6 node configuration has geometry of 2 triangles $45^{\circ}$ apart from each other. 8 node configuration IsoTruss is considered in the present study. 8 node configuration represents an IsoTruss with 8 longitudinal, 8 clockwise helical and 8 anticlockwise helical members as shown in the figure 1 . Cross sectional geometry of the 8 node configuration IsoTruss is having 2 squares $45^{\circ}$ apart 
from each other. Higher node configurations like 10 node, 12 node and so on has respective number of members. The Experimental axial loading characteristics of Isotruss are studied by Weaver Thomas J. etal.

\section{Material and Dimensions of IsoTruss}

$12 \mathrm{k}$ Carbon Fiber impregnated with epoxy resin is used as the material for the analysis of IsoTruss. The properties of material are enlisted in Table 1.

Table 1 Properties of the Material

\begin{tabular}{|c|c|}
\hline Modulus of Elasticity (GPa) & 120 \\
\hline Density (kg/cubic meter) & 1500 \\
\hline Cordage diameter $(\mathrm{mm})$ & 2 \\
\hline
\end{tabular}

IsoTruss considered for analysis is 8 node configuration having outer diameter of $70 \mathrm{~mm}$ and total length of $1.5 \mathrm{~m}$. IsoTruss ranging from bay length of $20 \mathrm{~mm}$ to $300 \mathrm{~m}$ is analyzed in the present study.

\section{Finite Element Modeling and Analysis}

Two types of 8 node configuration are considered for analysis:-

1) IsoTruss having internal longitudinal members only abbreviated as IT_IL (IsoTrus_Internal Longitudinal).

2) IsoTruss having internal and external longitudinal members abbreviated as IT_IEL (IsoTruss_Internal External Longitudinal).
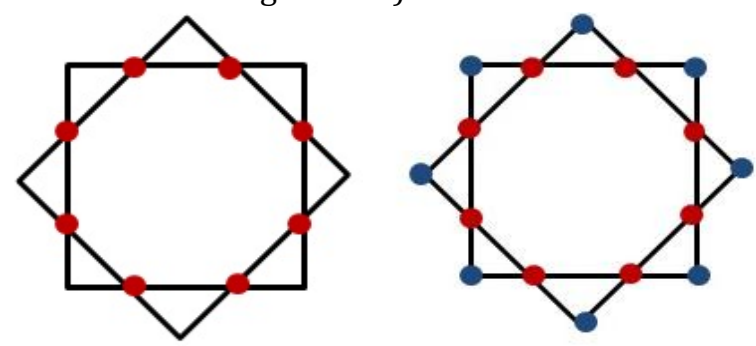

Fig. 2 Cross Sectional view of IT_IL(left ) and IT_IEL(right) indicating positions of longitudinal members.

IsoTruss is modeled and analyzed in the Finite Element Analysis Software Abaqus 6.12. The Abaqus input modeling file is encoded using MATLAB 7.0.1 programming software.

IT_IEL has 2 types of longitudinal member for handling the axial loads and thus it has an advantage over IT_IL under tensile loading. Weight of IT_IEL is slight greater than IT_IL due to an addition external longitudinal member.

\section{Results and Discussion}

The analysis of revels that the IT_IEL exhibits higher tensile load carrying capacity than IT_IL as shown in the Fig. 3. The maximum stress are developed in the longitudinal members or IT-IEL and IT_IL as they are aligned in the axial direction. Fig. 4 and 5 illustrates the stress development on the IsoTruss IT_IL and IT_IEL respectively for $100 \mathrm{~mm}$ bay length. The The tensile strength varies slightly with respect to the bay length.

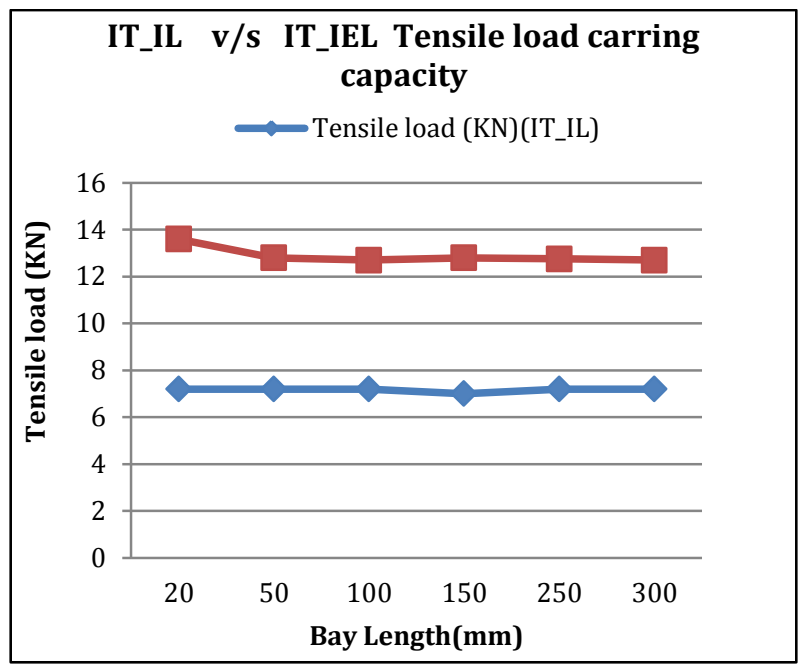

Fig. 3 Tensile Load carrying capacity for IT_IEL and IT_IL
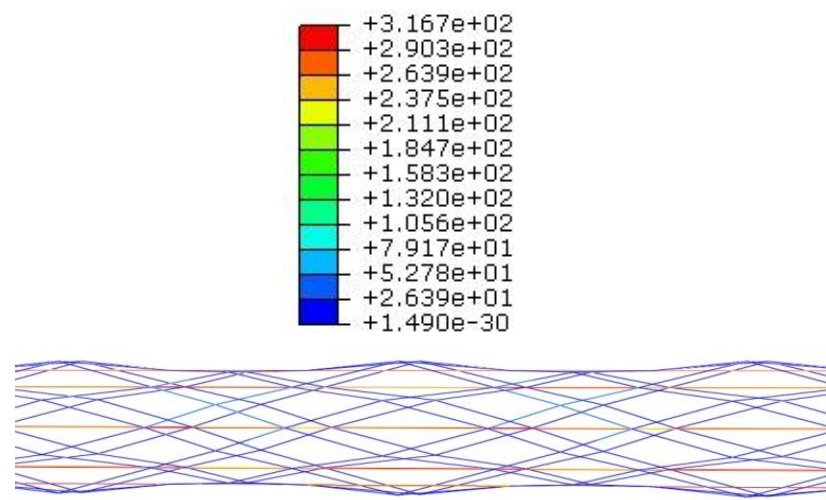

Fig.4 Variation of Stress(MPa) developed in IsoTruss IT_IL for $100 \mathrm{~mm}$ bay length

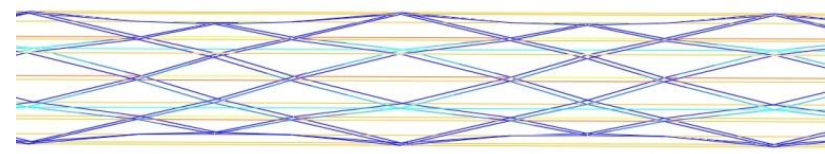

Fig. 5 Variation of Stress(MPa) developed in Isotruss IT-IEL for $100 \mathrm{~m}$ bay length.

Strength to weight ratio for the IsoTruss is presented in fig. 6. The strength to weight ratio gradually increases as the bay length increases from $20 \mathrm{~mm}$ to 
$100 \mathrm{~mm}$. For bay length $100 \mathrm{~mm}$ to $300 \mathrm{~mm}$ the strength to weight ratio remains approximately constant. Strength to weight ratio for IT-IEL is higher than IT-IL for every bay length as shown in the fig. 6 .

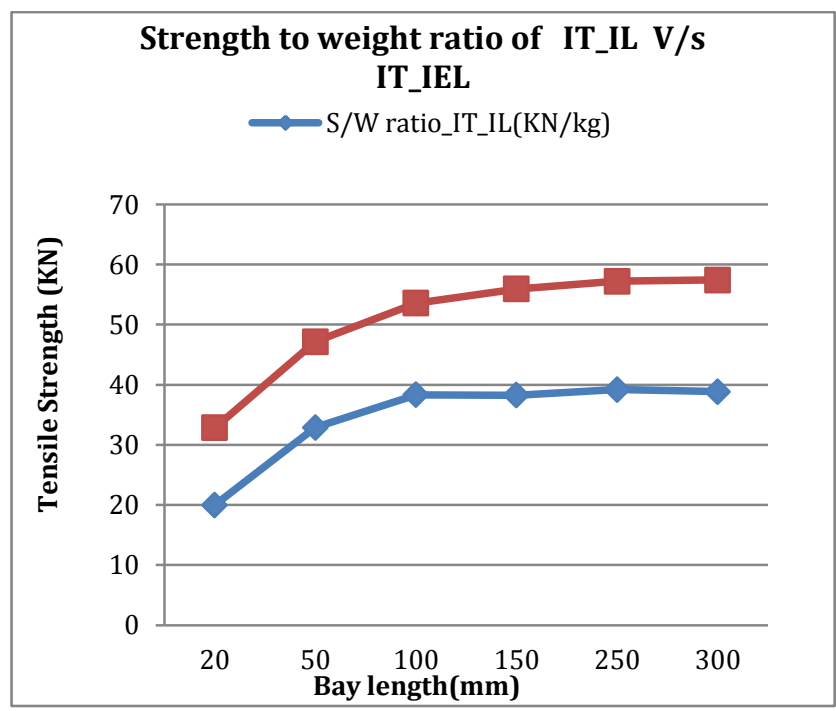

Fig.6 Strength to weight ratio for IT_IL and IT_IEL

\section{Conclusions}

Tensile load carrying capacity for IT_IEL is better than IT_IL. The tensile load Carrying capacity remained constant for the bay length $20 \mathrm{~mm}$ to $300 \mathrm{~mm}$.
Strength to weight ratio for IT_IEL and IT_IL varies over a range of $20 \mathrm{~mm}$ to $100 \mathrm{~m}$ bay length. Strength to weight ratio for IT_IEL is greater than IT_IL for bay length $20 \mathrm{~mm}$ to $300 \mathrm{~mm}$.

\section{References}

Weaver Thomas J., (1999), Mechanical characterization of a graphite/epoxy IsoTruss subject to simple and biaxial loads, M.S. Thesis, Brigham Young University, Provo, Utah.

Yoder Jacob A., (2000), IsoTruss to IsoTruss connections and small scale IsoTruss design for mountain bike frames, $\mathrm{S}$. Thesis, Brigham Young University, Provo, Utah.

Jones Layne S., (2000) Flexural behaviour of spirally consolidated double IsoTruss reinforced concrete beam, M.S. Thesis, Brigham Young University, Provo, Utah.

Jarvis David, (2001), Development of a rectangular IsoTruss for reinforced concrete beam, M.S. Thesis, Brigham Young University, Provo, Utah.

Phillips Joseph K, (2001), IsoTruss bicycle frame design using beam theory and shear flow analysis, M.S. Thesis, Brigham Young University, Provo, Utah.

Rackliffe Mary E, (2002), Development of ultra-lightweight IsoTruss grid structures, M.S. Thesis, Brigham Young University, Provo, Utah.

Mary E. Racliffe, David W. Jesen, Warren K. Lucas, (2005), Local and global buckling of ultra-light weight IsoTruss structures, Composites Science and Technology, Vol. 66, No. 5, pp. 282-288.s 